\title{
Relación entre el cumplimiento de las recomendaciones de actividad física y de sueño y el exceso de peso en escolares de la ciudad de Temuco, Chile

\author{
Relationship between physical activity and sleep \\ recommendations compliance and excess weight among school \\ children from Temuco, Chile
}

\author{
Mag. Javiera Ceppi-Larrain ${ }^{a}$, Mag. Damián Chandía-Poblete ${ }^{b, c}$, \\ Dr. Nicolás Aguilar-Farías ${ }^{a, c}$ y Dr. Jaime Cárcamo-Oyarzún $n^{a, c}$
}

a. Departamento de Educación Física, Deportes y Recreación, Universidad de La Frontera, Temuco, Chile.

b. School of Public Health and Social Work, Queensland University of Technology, Queensland, Australia.

c. Grupo de Investigación UFRO Actívate, Universidad de La Frontera, Temuco, Chile.

Correspondencia: Dr. Jaime CárcamoOyarzún: jaime.carcamo@ ufrontera.cl

Financiamiento: ANID Chile, a través de los proyectos FONDECYT 111160720 y FONDECYT 11170525.

Universidad de La Frontera. Programa InES19 Investigadores Libres.

Código FRO19101.

Conflicto de intereses: Ninguno que declarar.

Recibido: 3-12-2020

Aceptado: 2-5-2021

\section{RESUMEN}

Introducción. Dada la importancia que la actividad física y el sueño han adquirido para la salud, se han propuesto diversas recomendaciones para la población infantil. El propósito de este estudio fue determinar cómo se asocia el cumplimiento delas recomendaciones de actividad física y de sueño con el exceso de peso. Población y métodos. Para la medición de la actividad física y el sueño, se utilizaron acelerómetros ActiGraph wGT3X-BT ${ }^{\circledR}$. Se determinó el índice de masa corporal para las categorizaciones de ausencia (peso normal) o presencia (sobrepeso-obesidad)deexceso de peso. Resultados. Participaron 183 escolares (54,1\% eran niñas, edad $\mathrm{M}=10,95 \pm 1,07)$. El 85,4 \% no cumple con las recomendaciones de actividad física, mientras que el $75,6 \%$ no cumple con las recomendaciones de sueño. Los niños se diferencian significativamente de las niñas en tiempo de actividades físicas moderadas y vigorosas $(p=0,002)$, mas no así en cantidad de sueño. Los escolares con exceso de peso presentan menos tiempo de actividades de intensidad moderada y vigorosa $(p=0,004)$ y una menor cantidad de sueño $(p=0,010)$ que los escolares de peso normal. El no cumplir las recomendaciones de actividad física se asociaría con el exceso de peso (razón de momios [OR, por su sigla en inglés $]=8,178$; intervalo de confianza de1 95\% [IC95\%]: 1,465-45,635; $p=0,017$ ), lo que no se observó en las recomendaciones de sueño. Conclusiones. Un alto porcentaje de escolares no cumplen con las recomendaciones de actividad física y cantidad de sueño. Se observaron asociaciones según el sexo y la presencia de exceso de peso. Es necesario desarrollar estrategias para fomentar el cumplimiento de estas recomendaciones en la población infantil. Palabras clave: actividad física, sueño, sobrepeso, obesidad, acelerometría.

http:/ / dx.doi.org/10.5546/ aap.2021.370 Texto completo en inglés:

http: / / dx.doi.org/10.5546/ aap.2021.eng.370
Cómo citar: Ceppi-Larraín J, Chandía-Poblete D, Aguilar-Farías N, Cárcamo-Oyarzún J. Relación entre el cumplimiento de las recomendaciones de actividad física y de sueño y el exceso de peso en escolares de la ciudad de Temuco, Chile. Arch Argent Pediatr 2021;119(6):370-377.

\section{INTRODUCCIÓN}

La prevalencia de exceso de peso en la población infantil se ha incrementado ostensiblemente en las últimas décadas ${ }^{1}$ y es considerada una epidemia de salud pública en todo el mundo ${ }^{2}$ debido a las enfermedades degenerativas que la acompañan. ${ }^{3}$

Diversos estudios indican que la inactividad física sería uno de los principales factores que contribuyen a la prevalencia de exceso de peso. ${ }^{4}$ Esta situación se torna muy preocupante, ya que la inactividad física y la conducta sedentaria predominan en la mayoría de los países, ${ }^{5}$ y es común que los escolares pasen largos períodos frente a pantallas, en contraste con el poco tiempo dedicado a los juegos activos. ${ }^{6}$ La actividad física regular ofrece múltiples beneficios en la población infantil. ${ }^{7}$ Además de reducir el riesgo de obesidad, ${ }^{8}$ mejora la salud psicológica, aumenta los niveles de autoestima ${ }^{9} \mathrm{y}$ disminuye los niveles de estrés. ${ }^{10}$ Asimismo, colabora con el fomento del desarrollo de la competencia social percibida, ${ }^{11}$ mejora la maduración del sistema nervioso, ${ }^{12}$ contribuye al fomento de la competencia motriz ${ }^{13}$ y mejora el rendimiento escolar. ${ }^{14}$ 
Otro de los factores emergentes que ha sido relacionado con el exceso de peso es la cantidad de sueño. ${ }^{15,16}$ Se ha observado que, cuanto más larga y estable es la cantidad del sueño, es menos probable que un niño manifieste disfunciones metabólicas. ${ }^{17}$ En las últimas décadas, los hábitos de sueño de los niños han cambiado de manera negativa en relación con generaciones previas: se observa una marcada disminución de la cantidad de sueño. ${ }^{18}$

Dada la importancia que tanto la actividad física como la cantidad de sueño han adquirido para la salud y el bienestar infantil, se han propuesto diversas recomendaciones para cumplir con un día saludable ( 24 horas) que comprende una combinación de la cantidad de sueño, los comportamientos sedentarios (tiempo de pantalla) y la actividad física de intensidad ligera, moderada y vigorosa. ${ }^{19-21}$ La evidencia en la población escolar chilena indicaría que existe un bajo cumplimiento de las recomendaciones de sueño, ${ }^{22}$ de tiempo de pantalla ${ }^{23} \mathrm{y}$ de actividad física. ${ }^{24}$ Los niños y adultos que no cumplen con las recomendaciones diarias de actividad física y sueño presentarían mayor riesgo de sufrir exceso de peso. ${ }^{25}$ Considerando la alta incidencia de exceso de peso en escolares chilenos ${ }^{26}$ y los niveles elevados de inactividad física, ${ }^{24}$ el presente estudio tiene como propósito determinar la asociación entre el cumplimiento de las recomendaciones de actividad física y de cantidad de sueño con el exceso de peso en escolares de Temuco, Chile.

\section{POBLACIÓN Y MÉTODOS}

Se utilizó un muestreo no probabilístico por conveniencia, en donde participaron estudiantes de $5 .^{\circ}$ y $6 .^{\circ}$ de primaria de cinco centros educativos de distintos niveles socioeconómicos y ubicaciones geográficas de la ciudad de Temuco, Chile. El criterio de inclusión fue estar cursando $5 .^{\circ} \mathrm{o} 6 .^{\circ}$ de primaria y se eliminaron los escolares que no completaron las evaluaciones de forma íntegra (antropométrica y acelerometría).

Para el cálculo del tamaño muestral, se consideraron los siguientes datos: a) 3,7\% de niños y niñas activos según acelerometría, en un estudio previo. ${ }^{22}$ b) tamaño de 20 participantes por conglomerado (curso), c) $5 \%$ de nivel de significancia, d) precisión absoluta de $5 \%$, y e) tamaño de efecto de 1,3 basado en Williamson y cols. ${ }^{27}$ Con esto se estimó un mínimo de 200 participantes (10 conglomerados o cursos). Con la estimación de un porcentaje de respuesta del $80 \%$, se calculó una muestra final de 240 participantes.

El protocolo del estudio contó con la aprobación del Comité Ético de la Universidad de La Frontera (acta N. $\left.{ }^{\circ} 122 \_17\right)$. Los padres firmaron un consentimiento y los escolares dieron su consentimiento escrito para participar de manera voluntaria del estudio.

\section{Procedimientos}

Los participantes del estudio fueron evaluados por personal capacitado, en sus respectivos centros educativos, en el horario de clases de Educación Física, y completaron un cuestionario en donde debían ingresar la información sociodemográfica. Posteriormente, se realizaron las evaluaciones de peso y talla. Por último, se instruyó a los escolares sobre el uso de los acelerómetros, para luego hacer entrega de estos y verificar su ubicación correcta.

\section{Actividad física y cantidad de sueño}

Para la medición de la actividad física, la conducta sedentaria y el sueño, se utilizaron acelerómetros ActiGraph wGT3X-BT ${ }^{\boxplus}$ (ActiGraph, Pensacola, FL), los que fueron adquiridos a través del proyecto Fondecyt 11170525, financiado por la Agencia Nacional de Investigación de Chile. A los participantes se les solicitó que usaran el acelerómetro durante 7 días de forma ininterrumpida ${ }^{28}$ y se les recomendó quitárselo solo para actividades que involucraran agua (por ejemplo, ducharse). El acelerómetro se ubicaba en el lado derecho, a nivel de la cintura, a nivel de la línea medio-axilar y a la altura de la cresta ilíaca.

La descarga de datos de los acelerómetros se realizó en tiempo de almacenamiento (epochs) de 60 segundos con el software Actilife 6.13.4 ${ }^{\circledR}$ (Pensacola, FL, USA). Para el presente estudio se utilizaron los datos de tiempo sedentario, tiempo en actividad física ligera, tiempo en actividad física moderada y tiempo en actividad física vigorosa, expresados en minutos.

Primero, se detectó el tiempo de no uso del acelerómetro. La cantidad mínima de datos que se consideró como válida fue de 4 días con al menos 10 horas de uso por día, incluido al menos un día de fin de semana. Posteriormente, se estimó la cantidad (tiempo) de sueño y el número de despertares por noche en todos los días hábiles a través del algoritmo de Sadeh. ${ }^{29}$ Sin considerar los datos de no uso del acelerómetro y de sueño, se realizó el análisis para detectar los tiempos en conducta sedentaria y en actividad 
física de distintas intensidades utilizando los puntos de corte de Evenson. ${ }^{30}$ Para clasificar un participante como activo físicamente, se utilizó como criterio el que haya tenido en promedio 60 minutos de actividad física moderada a vigorosa ( 3 a 5 equivalentes metabólicos [MET, por su sigla en inglés]) por día. ${ }^{21}$ Por otra parte, los participantes fueron clasificados en la categoría de cumplimiento de las recomendaciones de sueño si habían dormido entre 9 y 11 horas diarias en promedio, según las pautas canadienses. ${ }^{19}$

\section{Estado nutricional}

Para la evaluación del peso, se utilizó una balanza marca Tanita ${ }^{a}$, modelo UM2204 (precisión de 0,2 kg, capacidad máxima $136 \mathrm{~kg}$ ). Se midió con los pies descalzos, pantalón de buzo y remera. La estatura se tomó con un estadiómetro Seca ${ }^{\circledR}$ modelo 217 (precisión de $1 \mathrm{~mm}$ ). La presencia o ausencia de exceso de peso se determinó a través del índice de masa corporal (IMC), considerando los parámetros específicos para cada edad y sexo según la Organización Mundial de la Salud. ${ }^{31}$ La clasificación normopeso (percentil < 85) fue categorizada como "ausencia de exceso de peso", mientras que las clasificaciones sobrepeso y obeso se categorizaron como "presencia de exceso de peso" (sobrepeso: IMC en percentil $\geq 85$ a $<97$; obesidad: IMC en percentil $\geq 97$ ).

\section{Análisis estadístico}

Se calcularon estadísticos descriptivos (medidas de tendencia central y frecuencias absolutas y relativas). Se empleó la prueba de Kolmogorov-Smirnov para conocer si las variables siguieron la distribución normal. Para comparar las variables de actividad física y sueño según el sexo de los escolares y el cumplimiento de las recomendaciones según la ausencia o presencia de exceso de peso, se utilizó la prueba $t$ de Student o U de Mann-Whitney, según el caso. Para determinar la asociación entre el cumplimiento de las recomendaciones de actividad física y sueño con la presencia de exceso de peso, se realizó un análisis de regresión logística, ajustado a edad, sexo y tiempo en conducta sedentaria. Se consideró como nivel de significancia $p<0,05$. El análisis estadístico se realizó con el software IBM SPSS $25^{\circledR}$.

\section{RESULTADOS}

En total 183 escolares participaron en el estudio (Figura 1), de los cuales el 54,1\% fueron a niñas; y de edades entre 9 y 13 años (media $[\mathrm{M}]=10,95 ; \pm 1,07$ ). Un $45,9 \%$ presentó la condición de normopeso (el 49,4 \% de las niñas; y el $41,7 \%$ de los niños) y el $54,1 \%$ presentó la condición de exceso de peso (el 50,6 \% de las niñas; y el 58,3 \% de los niños). En la Tabla 1 se muestran las características antropométricas, según el sexo de los escolares. No se encontraron diferencias significativas en la edad ni en las características antropométricas. En la Tabla 2 se exponen los resultados descriptivos de actividad física y sueño de la muestra en función del sexo. Se encontraron diferencias en la actividad

FIGURA 1. Flujograma de los participantes del estudio

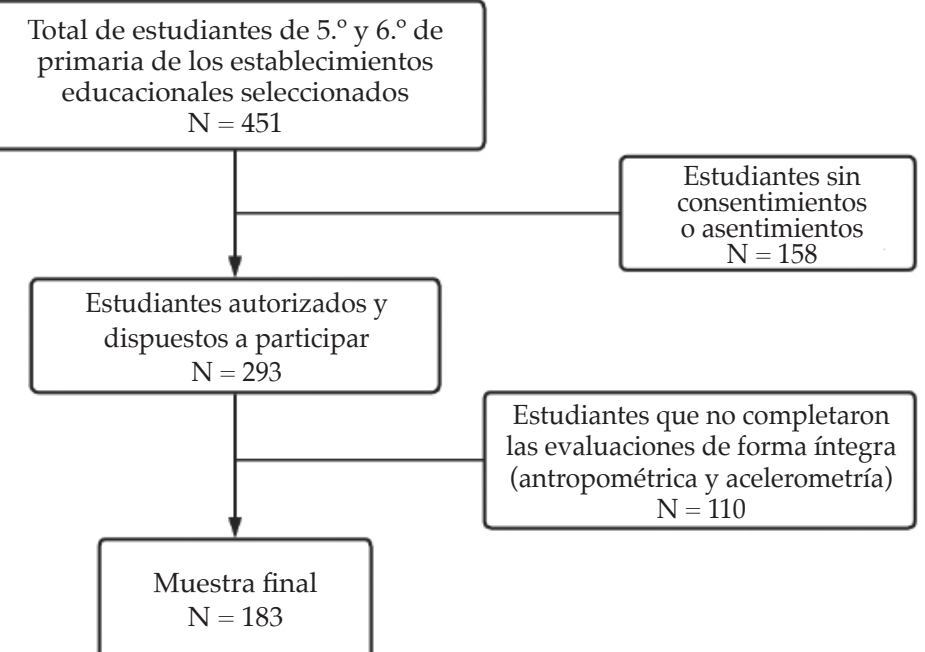


física moderada $(p=0,001)$ y en la suma de las actividades físicas moderada y vigorosa $(p=0,002)$, en donde los niños evidenciaron ser más activos que las niñas. En las características de sueño no se hallaron diferencias significativas.
En la Tabla 3 se analizan las frecuencias de los escolares que cumplieron las recomendaciones de actividad física moderada y vigorosa, de la cantidad de sueño, y de ambas. Es posible observar que fue baja la proporción de escolares

Tabla 1. Características demográficas según sexo en una muestra de escolares de la ciudad de Temuco, Chile $(n=183)$

\begin{tabular}{|c|c|c|c|c|c|c|c|}
\hline & & & \multicolumn{4}{|c|}{ Sexo } & \\
\hline & \multicolumn{2}{|c|}{$\begin{array}{c}\text { Total } \\
(\mathrm{n}=183)\end{array}$} & \multicolumn{2}{|c|}{$\begin{array}{c}\text { Niñas } \\
(\mathrm{n}=99)\end{array}$} & \multicolumn{2}{|c|}{$\begin{array}{c}\text { Niños } \\
(n=84)\end{array}$} & \multirow[b]{2}{*}{$p$} \\
\hline & $\mathbf{M}$ & DE & $\mathbf{M}$ & DE & $\mathbf{M}$ & DE & \\
\hline Edad (años) & 11,0 & 1,1 & 10,8 & 0,6 & 11,1 & 1,1 & 0,138 \\
\hline Peso (kg) & 45,2 & 10,2 & 45,4 & 10,6 & 45,0 & 9,9 & 0,823 \\
\hline Talla $(\mathrm{cm})$ & 145,0 & 7,3 & 145,5 & 7,3 & 144,4 & 7,4 & 0,390 \\
\hline IMC & 21,4 & 3,8 & 21,3 & 3,9 & 21,4 & 3,6 & 0,851 \\
\hline
\end{tabular}

IMC: índice de masa corporal.

TABla 2. Datos descriptivos de actividad física y sueño según sexo en una muestra de escolares de la ciudad de Temuco, Chile $(n=183)$

\begin{tabular}{|c|c|c|c|c|c|c|c|}
\hline & & & \multicolumn{4}{|c|}{ Sexo } & \multirow[b]{3}{*}{$p$} \\
\hline & \multicolumn{2}{|c|}{$\begin{array}{c}\text { Total } \\
(\mathrm{n}=183)\end{array}$} & \multicolumn{2}{|c|}{$\begin{array}{c}\text { Niñas } \\
(\mathrm{n}=99)\end{array}$} & \multicolumn{2}{|c|}{$\begin{array}{l}\text { Niños } \\
(n=84)\end{array}$} & \\
\hline & $\mathbf{M}$ & $\mathrm{DE}$ & $\mathbf{M}$ & DE & $\mathbf{M}$ & $\mathrm{DE}$ & \\
\hline Conducta sedentaria (min/día) & 453,0 & 67,9 & 453,2 & 66,4 & 452,7 & 70,3 & 0,960 \\
\hline \multicolumn{8}{|l|}{ Actividad física (min/ día) } \\
\hline Liviana & 415,8 & 59,5 & 418,1 & 59,5 & 412,8 & 59,8 & 0,570 \\
\hline Moderada & 32,8 & 13,9 & 29,6 & 10,5 & 37,0 & 16,5 & 0,001 \\
\hline Vigorosa & 7,9 & 7,8 & 6,8 & 9,3 & 5,7 & 9,6 & 0,055 \\
\hline Moderada y vigorosa & 40,7 & 20,0 & 36,3 & 15,0 & 46,1 & 23,9 & 0,002 \\
\hline \multicolumn{8}{|l|}{ Sueño } \\
\hline Cantidad de sueño (min/ día) & 514,0 & 42,2 & 516,9 & 46,0 & 510,5 & 3,9 & 0,324 \\
\hline Despertares por noche (n) & 7,8 & 3,7 & 8,0 & 3,6 & 7,5 & 3,2 & 0,399 \\
\hline
\end{tabular}

Resultados expresados como media aritmética (M) desviación estándar (DE) y porcentajes.

Prueba $t$ de Student o U de Mann-Whitney según el caso. IMC: índice de masa corporal.

TABla 3. Cumplimiento de las recomendaciones de actividad física y cantidad de sueño medidas con acelerómetros en una muestra de escolares de 9 a 13 años de la ciudad de Temuco, Chile $(n=183)$

\begin{tabular}{|c|c|c|c|c|c|c|}
\hline & \multicolumn{2}{|c|}{ Total } & \multicolumn{4}{|c|}{ Según sexo } \\
\hline & \multirow[t]{2}{*}{$\mathbf{n}$} & \multirow[t]{2}{*}{$\%$} & \multicolumn{2}{|c|}{ Niñas } & \multicolumn{2}{|c|}{ Niños } \\
\hline & & & $\mathbf{n}$ & $\%$ & $\mathbf{n}$ & $\%$ \\
\hline \multicolumn{7}{|c|}{ Cumplimiento de recomendaciones de actividad física } \\
\hline No cumple & 140 & 85,4 & 84 & 92,3 & 56 & 76,7 \\
\hline Cumple & 24 & 14,6 & 7 & 7,7 & 17 & 23,3 \\
\hline \multicolumn{7}{|c|}{ Cumplimiento de recomendaciones de cantidad de sueño } \\
\hline No cumple & 124 & 75,6 & 64 & 71,1 & 60 & 81,1 \\
\hline Cumple & 40 & 24,4 & 26 & 28,9 & 14 & 18,9 \\
\hline \multicolumn{7}{|c|}{ Cumplimiento de ambas recomendaciones } \\
\hline No cumple ninguna & 123 & 68,3 & 68 & 68,6 & 55 & 67,0 \\
\hline Cumple 1 & 52 & 28,9 & 29 & 29,2 & 23 & 28,0 \\
\hline Cumple 2 & 6 & 2,8 & 2 & 2,2 & 4 & 5,0 \\
\hline
\end{tabular}


que cumplen las recomendaciones de actividad física moderada y vigorosa, y de sueño. El cumplimiento de ambas recomendaciones es ínfimo.

En la Tabla 4 se presentan las características antropométricas, de actividad física y de sueño de la muestra, según la presencia o ausencia de exceso de peso. Con relación a la actividad física, se observaron diferencias significativas en actividad moderada $(p=0,013)$ actividad vigorosa $(p=0,002)$ y en el total de actividad física moderada y vigorosa $(p=0,004)$; fueron más activos quienes no presentaban exceso de peso. En cuanto al sueño, se encontraron diferencias significativas en la cantidad de sueño $(p=0,010)$ : los estudiantes que no presentaron exceso de peso durmieron más que quienes presentaron esta condición.
Por último, el modelo de regresión logística mostró que los escolares que no cumplen la recomendación de actividad física moderada y vigorosa tienen mayor probabilidad de tener exceso de peso que aquellos que sí la cumplen $(\mathrm{OR}=8,178 ; p=0,017)$. No se observa asociación entre el cumplimiento de las recomendaciones de sueño y la probabilidad de tener exceso de peso (Tabla 5).

\section{DISCUSIÓN}

El presente estudio tuvo como propósito determinar la asociación entre el cumplimiento de las recomendaciones de actividad física y de cantidad de sueño, medidos con acelerómetros, con el exceso de peso en escolares de Temuco, Chile. En cuanto a los niveles de actividad física, el tiempo total de los participantes de este estudio

TABLA 4. Características antropométricas, de actividad física y sueño según exceso de peso en una muestra de escolares de la ciudad de Temuco, Chile $(n=183)$

\begin{tabular}{|c|c|c|c|c|c|}
\hline & \multicolumn{4}{|c|}{ Exceso de peso } & \multirow[b]{3}{*}{$p$} \\
\hline & \multicolumn{2}{|c|}{$\begin{array}{c}\text { Presencia } \\
(\mathrm{n}=69)\end{array}$} & \multicolumn{2}{|c|}{$\begin{array}{c}\text { Ausencia } \\
(\mathrm{n}=80)\end{array}$} & \\
\hline & $\mathbf{M}$ & DE & $\mathbf{M}$ & DE & \\
\hline Edad (años) & 10,9 & 0,63 & 11,1 & 0,66 & 0,152 \\
\hline Peso $(\mathrm{kg})$ & 51,3 & 9,24 & 38,2 & 5,79 & 0,000 \\
\hline Talla $(\mathrm{cm})$ & 145,7 & 7,59 & 144,2 & 6,98 & 0,229 \\
\hline IMC & 24,1 & 2,90 & 18,3 & 1,70 & 0,000 \\
\hline Conducta sedentaria (min/día) & 458,4 & 65,33 & 448,4 & 71,86 & 0,404 \\
\hline \multicolumn{6}{|l|}{ Actividad física (min/día) } \\
\hline Liviana & 418,3 & 59,67 & 413,2 & 60,39 & 0,627 \\
\hline Moderada & 29,1 & 10,67 & 34,8 & 14,84 & 0,013 \\
\hline Vigorosa & 5,3 & 5,52 & 9,2 & 8,41 & 0,002 \\
\hline Moderada y vigorosa & 34,4 & 13,56 & 43,8 & 22,01 & 0,004 \\
\hline \multicolumn{6}{|l|}{ Sueño } \\
\hline Cantidad de sueño ( $\mathrm{min} /$ noche) & 505,8 & 45,37 & 524,6 & 37,59 & 0,010 \\
\hline Despertares por noche $(\mathrm{n})$ & 4,0 & 0,46 & 3,7 & 0,43 & 0,105 \\
\hline
\end{tabular}

Resultados expresados como media aritmética $(\mathrm{M})$ y desviación estándar (DE).

Prueba $t$ de Student o U de Mann-Whitney según el caso. IMC: índice de masa corporal.

TABLA 5. Modelo ajustado de regresión logística que predice el exceso de peso según el cumplimiento de recomendaciones de actividad física y sueño

\begin{tabular}{lcccc}
\hline & OR & \multicolumn{2}{c}{ IC95 \% } & \multirow{2}{*}{$p$} \\
\cline { 3 - 4 } & & Inferior & Superior & 0,553 \\
Edad & & 0,441 & 1,550 & 0,068 \\
Sexo & 0,827 & 0,942 & 5,335 & 0,891 \\
Tiempo sedentario & 2,242 & 0,995 & 1,006 & 0,017 \\
Cumple recomendaciones de actividad física & 1,000 & 1,465 & 45,635 & 0,584 \\
Cumple recomendaciones de sueño & 8,178 & 0,512 & 3,287 & \\
\hline
\end{tabular}

Modelo ajustado por edad, sexo y tiempo en conducta sedentaria. OR: odds ratio; IC: intervalo de confianza. 
fue menor al observado en niños de similar edad de Canadá (58 min/ día) y de Australia (65 min/ día), pero cercano al registrado en India (48 min/ día) y en Brasil (44 min/ día). ${ }^{32}$ En relación con el sexo, los niños presentaron casi 10 minutos más de actividad física moderada y vigorosa que las niñas; esto coincide con diversos estudios que indican que los niños son más activos que las niñas. ${ }^{33,34}$ Estos resultados podrían explicarse en parte por factores biológicos, tales como la estructura de las células musculares inducida por las hormonas sexuales, ${ }^{35}$ así como también por las diferencias en la competencia motriz, en donde los niños evidencian mejores desempeños. ${ }^{36}$

En lo que respecta a la cantidad de sueño, los participantes presentaron una media de 8,5 horas diarias, lo que es menor a lo encontrado en niños de Australia (9,4 horas) y de Canadá $(9,1$ horas), pero similar a la cantidad de sueño de los escolares de Brasil ( 8,8 horas), de China $(8,5$ horas $)$ y de India $(8,6$ horas $) .{ }^{32}$ Las niñas mostraron valores levemente mayores que los niños, sin que esta diferencia sea estadísticamente significativa; lo cual coincide con los resultados de Toledo-Vargas, y cols. ${ }^{22}$ Otros estudios han reportado que los niños duermen más tiempo, ${ }^{37}$ por lo que el rol que juega el sexo en la cantidad de sueño en la población infantil aún no está del todo definido.

En nuestro estudio, solo el 14,6\% cumplió con las recomendaciones de actividad física. Es un bajo cumplimiento si se compara con niños y niñas de países como Australia (55\%), Brasil (44 \%) o Canadá (44\%), pero similar a lo observado en China (15\%). ${ }^{33}$ En cuanto a las recomendaciones de cantidad de sueño, solo un 24,4 \% las cumplió, proporción menor a la obtenida en otros estudios con escolares chilenos ${ }^{22,26}$ y a la observada en otros países como Australia $(75 \%)$ y Canadá $(58 \%)$, pero similar a la advertida en Brasil (24\%). ${ }^{32}$ Al evaluar el porcentaje de la muestra que cumplió ambas recomendaciones de actividad física y de sueño, la proporción es mucho menor: el 2,8\%. Estos niveles de cumplimiento extremadamente bajos se asimilan a los encontrados por Toledo-Vargas y cols. ${ }^{22}$ lo que debe colocar a Chile en una situación de alerta, ya que un mayor porcentaje de escolares tendría riesgo aumentado de presentar exceso de peso.

En referencia al análisis de los tiempos en actividad física y la cantidad de sueño según la presencia o no de exceso de peso, los escolares sin exceso de peso presentaron casi 9 minutos más de actividad física moderada y vigorosa que los escolares con exceso de peso, lo que va en línea con la evidencia encontrada en otros estudios. ${ }^{37,38}$ Asimismo, los escolares sin exceso de peso durmieron casi 20 minutos diarios más que los que tuvieron exceso de peso, resultados similares a los obtenidos por Ruiz y cols..$^{39}$ Estos resultados concuerdan con la tendencia emergente sobre la relación de la actividad física, la cantidad de sueño y el estado nutricional, que indica que, cuanto más larga y estable es la cantidad del sueño, es menos probable que un niño manifieste disfunciones metabólicas. ${ }^{17}$

Al evaluar la asociación entre el cumplimiento de las recomendaciones de actividad física moderada a vigorosa y de cantidad de sueño con la presencia de exceso de peso, los escolares que no cumplen la recomendación de actividad física tuvieron mayor probabilidad de tener exceso de peso que aquellos niños que sí la cumplen, similar a lo reportado por Roman-Viñas y cols. ${ }^{40}$ y Katzmarzyk y col..$^{25}$ Por otro lado, no se encontró asociación entre el cumplimiento de las recomendaciones de cantidad de sueño y la presencia de exceso de peso. A pesar de que en nuestro estudio se encontraron diferencias significativas en la cantidad de sueño entre los escolares de peso normal y con exceso de peso, una gran cantidad de los escolares (independientemente de la condición de peso) no cumplieron las recomendaciones de cantidad de sueño, lo que podría explicar esta falta de asociación, por lo que esta divergencia indica la necesidad de profundizar en esta temática.

Dentro de las fortalezas del estudio, se puede destacar el uso de equipos de acelerometría, que permiten una mayor precisión en la evaluación tanto de los niveles de actividad física como de la cantidad de sueño. Por otro lado, dentro de las limitaciones de este trabajo, se puede considerar que, teniendo en cuenta que el estudio es de tipo transversal, no es posible establecer la causalidad de estos resultados. De manera similar a lo observado en otro estudio conducido en la misma región de Chile, ${ }^{22}$ dos tercios de la muestra considerada originalmente completaron el protocolo para ser incluidos en el análisis final; sin embargo, no se alcanzó el mínimo de la muestra estimada. Otra limitación fue no tener un registro de los hábitos alimentarios de los estudiantes, lo que podría influir en los resultados. Asimismo, no se consideró una diferenciación de los días hábiles y los fines de semana, o la medición de distintos períodos del año, lo que podría aportar mayor 
información sobre el comportamiento tanto de actividad física como la cantidad del sueño de los escolares.

\section{CONCLUSIONES}

El cumplimiento de las recomendaciones de actividad física y de cantidad de sueño en la muestra estudiada es bajo. Los niños presentan más tiempo de actividad física moderada y vigorosa que las niñas, mientras que los estudiantes con exceso de peso muestran valores más bajos en estos niveles que sus pares que tienen un peso normal. En referencia a la cantidad de sueño, no se encontraron diferencias significativas según sexo, pero sí en lo que respecta al exceso de peso, ya que los estudiantes con esta condición duermen menos tiempo. El no cumplir las recomendaciones de actividad física se asociaría con el exceso de peso. No se encontró asociación entre el cumplimiento de las recomendaciones de cantidad de sueño y la presencia de exceso de peso.

\section{REFERENCIAS}

1. Broyles S, Katzmarzyk PT, Srinivasan SR, Chen W, et al. The pediatric obesity epidemic continues unabated in Bogalusa, Louisiana. Pediatrics. 2010; 125(5):900-5.

2. Kumar S, Kaufman T. Childhood obesity. Panminerva Med. 2018; 60(4):200-12.

3. Mönckeberg BF, Muzzo BS. La desconcertante epidemia de obesidad. Rev Chil Nutr. 2015; 42(1):96-102.

4. Myers A, Gibbons C, Finlayson G, Blundell J. Associations among sedentary and active behaviours, body fat and appetite dysregulation: investigating the myth of physical inactivity and obesity. Br J Sports Med. 2017; 51(21):1540-4.

5. Aguilar-Farias N, Martino-Fuentealba P, Carcamo-Oyarzun J, Cortinez-O'Ryan A, et al. A regional vision of physical activity, sedentary behaviour and physical education in adolescents from Latin America and the Caribbean: results from 26 countries. Int J Epidemiol. 2018; 47(3):976-86.

6. Berglind D, Tynelius P. Objectively measured physical activity patterns, sedentary time and parent-reported screen-time across the day in four-year-old Swedish children. BMC Public Health. 2018; 18(1):69.

7. Alvarez-Pitti J, Casajús Mallén J, Leis Trabazo R, Lucía A, et al. Ejercicio físico como «medicina» en enfermedades crónicas durante la infancia y la adolescencia. An Pediatr (Barc). 2020; 92(3):173.e1-8.

8. Ip P, Ho F, Louie L, Chung T, et al. Childhood obesity and physical activity-friendly school environments. J Pediatr. 2017; 191:110-6.

9. Delgado-Floody P, Caamaño-Navarrete F, Jerez-Mayorga D, Cofré-Lizama A, et al. Obesidad, autoestima y condición física en escolares. Rev Fac Med. 2017; 65(1):43-8.

10. Messerli-Bürgy N, Horsch A, Schindler C, Boichat A, et al. Influence of Acute Physical Activity on Stress Reactivity in Obese and Normal Weight Children: A Randomized Controlled Trial. Obes Facts. 2019; 12(1):115-30.

11. Bedard C, HannaS, Cairney J. A longitudinal study of sport participation and perceived social competence in youth. $J$ Adolesc Health. 2020; 66(3):352-9.

12. Bidzan-Bluma I, Lipowska M. Physical activity and cognitive functioning of children: a systematic review. Int J Environ Res Public Health. 2018; 15(4):800.

13. Barnett LM, Salmon J, Hesketh KD. More active pre-school children have better motor competence at school starting age: an observational cohort study. BMC Public Health. 2016; 16(1):1068.

14. Iglesias A, Planells E, Molina López J. Prevalencia de sobrepeso y obesidad, hábitos alimentarios y actividad física y su relación sobre el rendimiento académico. Retos. 2019; 36:167-73.

15. Chaput JP, Brunet M, Tremblay A. Relationship between short sleeping hours and childhood overweight/obesity: results from the 'Quebecen Forme' Project. Int JObes (Lond). 2006; 30(7):1080-5.

16. Knutson KL, Spiegel K, Penev P, Van Cauter E. The metabolic consequences of sleep deprivation. Sleep Med Rev. 2007; 11(3):163-78.

17. Spruyt K, Molfese DL, Gozal D. Sleep duration, sleep regularity, body weight, and metabolic homeostasis in school-aged children. Pediatrics. 2011; 127(2):e345-52.

18. Matricciani L, Olds T, Petkov J. In search of lost sleep: secular trends in the sleep time of school-aged children and adolescents. Sleep Med Rev. 2012; 16(3):203-11.

19. Tremblay M, Carson V, Chaput J, Connor Gorber S, et al. Canadian 24-hour movement guidelines for children and youth: an integration of physical activity, sedentary behaviour, and sleep. Appl Physiol Nutr Metab. 2016; 41(6 Suppl 3):S311-27.

20. Tremblay M, Carson V, Chaput J. Introduction to the Canadian 24-Hour Movement Guidelines for Children and Youth: An Integration of Physical Activity, Sedentary Behaviour, and Sleep. Appl Physiol Nutr Metab. 2016; 41(6 Suppl 3):iii-iv.

21. World Health Organization. WHO Guidelines on physical activity and sedentary behaviour, 2020. [Acceso: 03 de marzo de 2021]. Disponible en: https:/ / www.who.int/ publications / i/item/9789240015128

22. Toledo-Vargas M, Perez-Contreras P, Chandia-Poblete D, Aguilar-Farias N. Compliance of the 24-Hour Movement Guidelinesin 9-to 11-Year-Old Children From a Low-Income Town in Chile. J Phys Act Health. 2020; 17(10):1034-41.

23. Aguilar M, Vergara FA, Velásquez E, García-Hermoso A. Actividad física, tiempo de pantalla y patrones de sueño en niñas chilenas. An Pediatr (Barc). 2015; 83(5):304-10.

24. Aguilar-Farias N, Miranda-Marquez S, Sadarangani KP, Martino-Fuentealba P, et al. Results from Chile's 2018 report card on physical activity for children and youth. $J$ Phys Act Health. 2018; 15(Suppl 2):S331-2.

25. KatzmarzykPT,Staiano AE. Relationship between meeting 24-hour movement guidelines and cardiometabolic risk factors in children. J Phys Act Health. 2017; 14(10):779-84.

26. Duran AS, Fuentes de la CN, Vásquez QS, Cediel GG, et al. Relación entre estado nutricional y sueño en escolares de la comuna de San Miguel, Santiago, Chile. Rev Chil Nutr. 2012; 39(1):30-7.

27. Williamson DA, Champagne CM, Harsha DW, Han H, et al. Effect of an environmental school $\square$ based obesity prevention program on changes in body fat and body weight: a randomized trial. Obesity (Silver Spring). 2012; 20(8):1653-61.

28. Tudor-Locke C, Barreira T, Schuna J Jr. Comparison of step outputs for waist and wrist accelerometer attachment sites. Med Sci Sports Exerc. 2015; 47(4):839-42.

29. Sadeh A, Acebo C. The role of actigraphy in sleep medicine. Sleep Med Rev. 2002; 6(2):113-24.

30. Evenson K, Catellier D, Gill K, Ondrak K, et al. Calibration of two objective measures of physical activity for children. J Sports Sci. 2008; 26(14):1557-65. 
31. World Health Organization. Growth reference data for 5-19 years. BMI for age (5-19 years), 2013. [Acceso: 03 de marzo de 2021]. Disponible en: https:/ / www.who.int/ tools/growth-reference-data-for-5to19-years/indicators/ bmi-for-age

32. Thivel D, Tremblay M, Katzmarzyk P, Fogelholm M, et al. Associations between meeting combinations of 24hour movement recommendations and dietary patterns of children: A 12-country study. Prev Med. 2019; 118:159-65.

33. Glinkowska B, Glinkowski W. Association of sports and physical activity with obesity among teenagers in Poland. Int J Occup Med Environ Health. 2018; 31(6):771-82.

34. Gutierrez-Hervas A, Cortés-Castell E, Juste-Ruíz M, RizoBaeza M. ¿Qué variables influyen en el cumplimiento de las recomendaciones de actividad física en preescolares?. An Pediatr (Barc). 2020; 92(3):156-64.

35. Wang WY, Hsieh YL, Hsueh MC, Liu Y, etal. Accelerometermeasured physical activity and sedentary behavior patterns in Taiwanese adolescents. Int J Environ Res Public Health. 2019; 16(22):4392.
36. Carcamo-Oyarzun J, Estevan I, Herrmann C. Association between Actual and Perceived Motor Competence in School Children. Int J Environ Res Public Health. 2020; 17(10):3408.

37. Harrex H, Skeaff S, Black K, Davison B, et al. Sleep timing is associated with diet and physical activity levels in 9-11 $\square$ year $\square$ old children from Dunedin, New Zealand: the PEDALS study. J Sleep Res. 2018; 27(4):e12634.

38. PereiraS, KatzmarzykP, Gomes T, Borges A, et al. Profiling physical activity, diet, screen and sleep habits in Portuguese children. Nutrients. 2015; 7(6):4345-62.

39. Ruiz N, Rangel A, Rodríguez C, Rodríguez L, et al. Relación entre el déficit de sueño nocturno, el exceso de peso y las alteraciones metabólicas en adolescentes. Arch Argent Pediatr. 2014; 112(6):511-8.

40. Roman-Viñas B, Chaput JP, Katzmarzyk PT, Fogelholm $\mathrm{M}$, et al. Proportion of children meeting recommendations for 24-hour movement guidelines and associations with adiposity in a 12-country study. Int J Behav Nutr Phys Act. 2016; 13(1):123.

\section{Archivos hace 75 años}

TRATAMIENTO AMBULATORIO DE LA GONOCOCCIA INFANTIL. CON PENICIIINA POR VIA ORAI. mes $\mathrm{m}$

Phor. DE. ALREDDO WIEDERIEOLD, DE. ANIONIETA MENDEL

It ota exposicicin mo queremos pecgonar una terapkutica deter

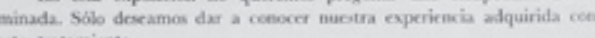
ete tratamicato.

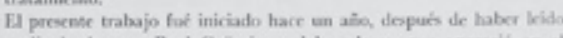
un ofudio hecho por Paul Györgi y colaboradores, qux apareció en et -Journal" del 17 de marso de 1945 , de una insinuarión hercha por c Prof. Ibarra L.

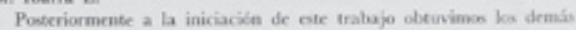
taeces bibliograficos.

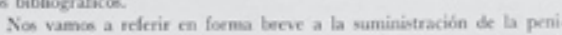

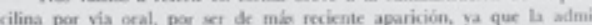

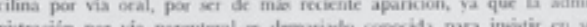

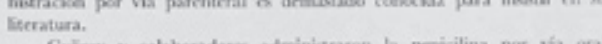

Gyăngy y colaboradoros admániseraron la penicilina por via oral

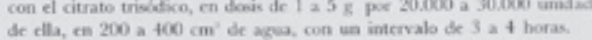

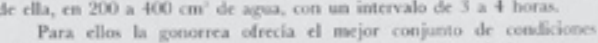
para la apecciacicen teraptivica de la penicilina por vía oral. Se trataror 15 adultos y 5 niños, ta maycria rosibentos a los sulfaporparsdos $\mathrm{L}$. doeis para los nithos fué de 10.000 anidades, cada 364 horas duraes. 2 a 3 dias, en combinación con $5 \mathrm{~g}$ de cinrano de sodio por dosib. Ent todos cotos cous $x$ obtuvo la curación en I a 3 diax

Estos autoes han scmetido a tratamirnte con pericilins por sia oral, otras afecciono: cems ostecriclitis etc.

Les autores va mencionatos creen que th cantidad de prenicilina y citrato de sodio, lo mikmo que el tipo de sal, podrias en el futuso, varia

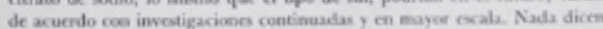

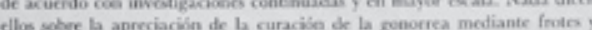

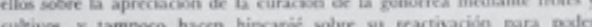

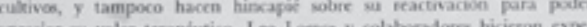

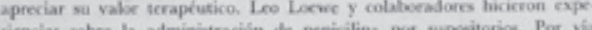

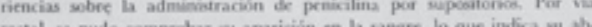

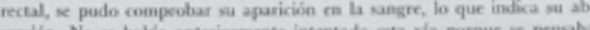
sorcion. No $x$ habix anteriormente intentado cta via porque se pernsabu 